\title{
New additions to Turkish Hyaloscyphaceae
}

\author{
Yasin UZUN ${ }^{1}$, Abdullah KAYA ${ }^{1}$, İbrahim Halil KARACAN², Semiha YAKAR' \\ ${ }^{1}$ Karamanoğlu Mehmetbey University, Science Faculty, Department of Biology, 70100 \\ Karaman, Turkey \\ ${ }^{2}$ Ömer Özmimar Religious Anatolian High School, 27220, Gaziantep, Turkey
}

\begin{abstract}
Five hyaloscyphaceous macrofungi taxa, Calycina conorum (Rehm) Baral, Discocistella grevillei (Berk.) Svrček, Hyalopeziza millepunctata (Lib.) Raitv., Lasiobelonium variegatum (Fuckel) Raitv. and Rodwayella citrinula (P. Karst.) Spooner, were given as new records for the mycobiota of Turkey. Four of them are the first members of the genera Calycina Nees ex Gray, Discocistella Svrček, Hyalopeziza Fuckel and Rodwayella Spooner in Turkey. The taxa are described briefly and the photographs related to their macro and micromorphologies are provided.
\end{abstract}

Key words: Macrofungi, New records, Gaziantep, Turkey.

\section{Türkiye için Hyaloscyphaceae'ye Yeni İlaveler}

Öz: Beş hyaloscyphaceous makromantar taksonu, Calycina conorum (Rehm) Baral, Discocistella grevillei (Berk.) Svrček, Hyalopeziza millepunctata (Lib.) Raitv., Lasiobelonium variegatum (Fuckel) Raitv. and Rodwayella citrinula (P. Karst.) Spooner, Türkiye mikobiyotası için yeni kayıt olarak verilmiştir. Bunlardan dört tanesi Calycina Nees ex Gray, Discocistella Svrček, Hyalopeziza Fuckel and Rodwayella Spooner cinslerinin Türkiye'deki ilk üyeleridir. Taksonların kısa betimlemesi yapılmış ve türlerin makro ve mikromorfolojilerine ait fotoğrafları verilmiştir.

Anahtar kelimeler: Makromantarlar, Yeni kayıtlar, Gaziantep, Türkiye.

\section{Introduction}

Hyaloscyphaceae Nannf. is a fungal family within the order Helotiales (Cannon and Kirk, 2007). The members of the family are mainly characterized by small apothecia covered with well differentiated marginal and lateral hairs. Species in this family are saprobic and generally have a cosmopolitan distribution (Han et al., 2014).

So far 10 taxa of Hyaloscyphaceae belonging to the genera Cistella Quél., Dasyscyphella Tranzschel, Lachnellula P. Karst., Lasiobelonium Ellis \& Everh., Neodasyscypha Suková \& Spooner and Perrotia Boud. have been recorded from Turkey (Sümer, 1982; Kaşık et al., 2002; Doğan and Öztürk,
2006; Akata et al., 2009a; Akata et al., 2009b; Alkan et al., 2010; Öztürk et al., 2010; Sesli and Denchev, 2014; Kaya et al., 2015; Uzun et al., 2015).

The present paper deals with the hyaloscyphaceous species collected from Gaziantep province and aims to make a contribution to the mycobiota of Turkey.

\section{Materials and methods}

Specimens were collected from various localities within the boundaries of Gaziantep province between 2014 and 2015. They were photographed in their natural habitats and ecological and morphological characteristics of them were recorded. 
Micromorphological studies were carried out under Nikon Eclipse $\mathrm{Ci}$ trinocular light microscope. With the help of the relevant literature (Raitviir, 1980; Breitenbach and Kränzlin, 1984; Raitviir and Galán, 1993; Cheype, 2004; Hairaud, 2010; Han et al., 2014; Friebes and Wendelin, 2015) they were idendified. The collected materials are deposited in Karamanoğlu Mehmetbey University, Kamil Özdağ Science Faculty, Department of Biology.

\section{Results}

The systematics of the taxa are given in accordance with Cannon and Kirk (2007), Kirk et al. (2008), and the Index Fungorum (www.indexfungorum.org; accessed 9 December 2016). The taxa are presented in alphabetical order with a brief description, habitat, locality, collection date, and accession numbers.
Leotiomycetes O.E. Erikss. \& Winka

Helotiales Nannf.

Hyaloscyphaceae Nannf.

Calycina conorum (Rehm) Baral

Syn: [Cystopezizella conorum (Rehm) Svrček, Pezizella conorum Rehm, Pezizella conorum Rehm var. conorum]

Macroscopic and microscopic features: Apothecia 0.2-0.6 mm in diameter, disc shaped, sessile, concrescent, ivory white to creamy yellow. Hymenial surface smooth, concolorous with the outside (Figure 1a). Asci 45-50 × 5-9 $\mu \mathrm{m}$, cylindrical. Paraphyses irregularly cylindrical, often tapering towards the apex (Figure 1b). Ascospores 7-8 × 2,5-3 $\mu \mathrm{m}$, smooth, hyaline, without guttules (Figure 1c).

Specimen examined: Gaziantep -Islahiye, Kozdere village, pine forest, on Pinus brutia Ten. cones, $37^{\circ} 06^{\prime} \mathrm{N}-36^{\circ} 39 \mathrm{E}, 560 \mathrm{~m}$, 15.03.2014, K.8617.

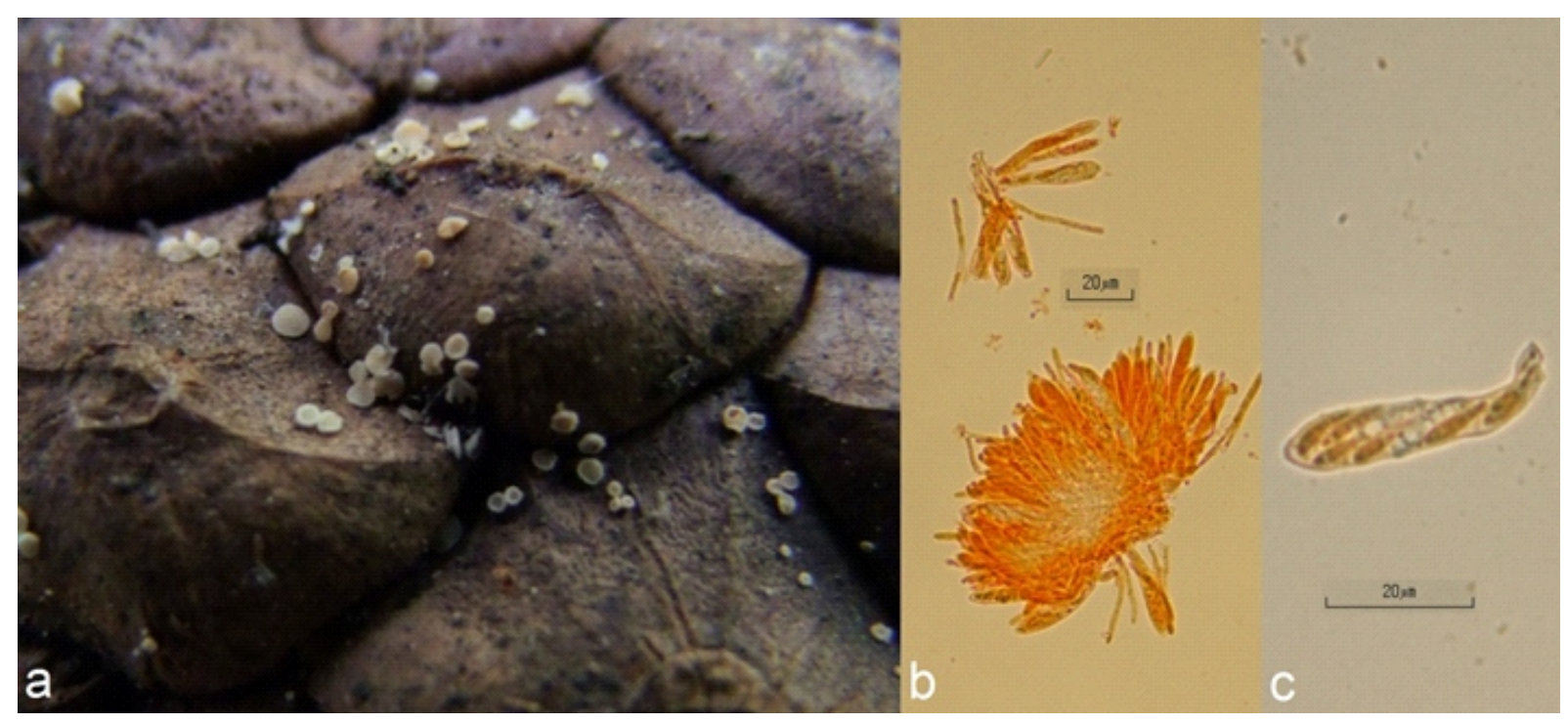

Figure 1. Calycina conorum: a. ascocarps, b. asci and paraphyses, c. ascospores in an ascus 
Discocistella grevillei (Berk.) Svrček

Syn: [Calloria coniicola Cooke \& W. Phillips, Cistella grevillei (Berk.) Raitv., Clavidisculum grevillei (Berk.) Raitv., Dasyscyphus berkeleyi (A. Bloxam) Massee, Dasyscyphus grevillei (Berk.) Massee, Lachnella berkeleyi (A. Bloxam) W. Phillips, Lachnum grevillei (Berk.) Nannf., Mollisia grevillei (Berk.) W. Phillips, Peziza berkeleyi A. Bloxam, Peziza grevillei Berk., Trichopeziza berkeleyi (A. Bloxam) Sacc., Trichopeziza grevillei (Berk.) Sacc., Urceolella berkeleyi (A. Bloxam) Boud.]

Macroscopic and microscopic features: Apothecia 0.3-0.6 mm in diameter, cup to soucer shaped, sessile, margin hairy, white, creamy white to light pinkish. Hymenial surface smooth, concolorous with the outside (Figure 2a). Asci 40-55 × 4,5-7 $\mu \mathrm{m}$, cylindrical. Paraphyses lanceolate (Figure 2b). Ascospores 7-9 × 1-2 $\mu \mathrm{m}$, cylindrical, smooth (Figure 2c).

\section{Specimen examined:}

Gaziantep-Yavuzeli, Halilbaşlı village, stream side, on dead Conium maculatum L. stem, $37^{\circ} 16^{\prime} \mathrm{N}-37^{\circ} 31^{\prime} \mathrm{E}, 560 \mathrm{~m}, 09.03 .2014$, K.8588; Sarıbuğdaylı village, stream side, on herbaceous stem, $37^{\circ} 17^{\prime} \mathrm{N}-37^{\circ} 31^{\prime} \mathrm{E}, 560 \mathrm{~m}$, 16.03.2014, K.8675.

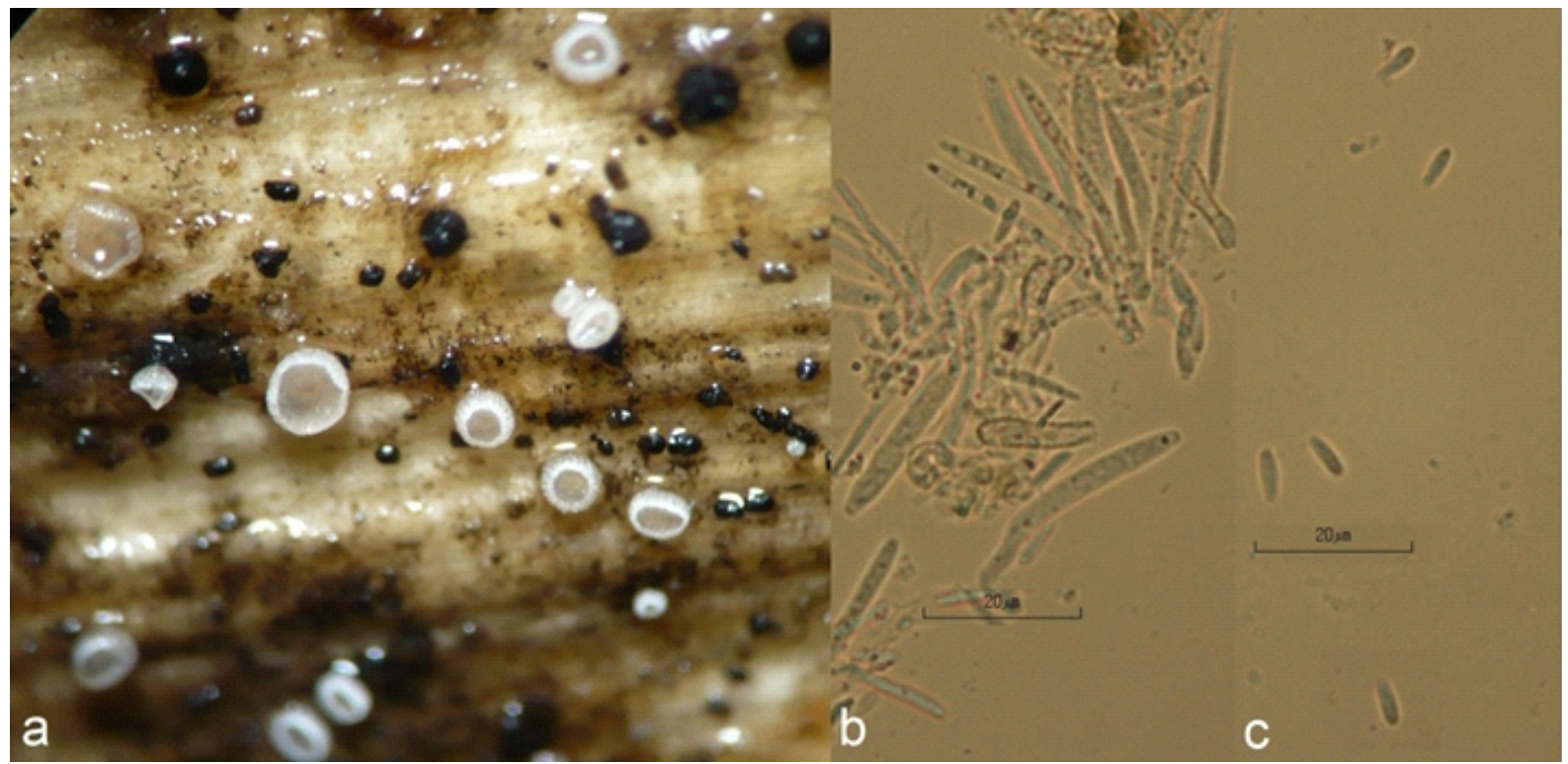

Figure 2. Discocistella grevillei: a. ascocarps, b. asci and paraphyses, c. ascospores

Hyalopeziza millepunctata (Lib.) Raitv.

Syn: [Dasyscyphus carmichaelii Massee, Dasyscyphus elaphines (Berk. \& Broome) Massee, Dasyscyphus scrupulosus (P. Karst.) Velen., Dasyscyphus scrupulosus var. melampyri Velen., Dasyscyphus scrupulosus (P. Karst.) Velen., var. scrupulosus, Dumontinia ulmariae (Velen.) Holst-Jensen, Helotium carmichaelii (Berk.) Massee, Helotium scrupulosum P. Karst., Hyalopeziza scrupulosa (P. Karst.) Raitv., Hyaloscypha millepunctata (Lib.) Boud., Hymenoscyphus carmichaelii Berk., Lachnella grisella Cooke \& W. Phillips, Lachnella scrupulosa (P. Karst.) W. Phillips, Mollisia elaphines (Berk. \& Broome) Gillet, Mollisia millepunctata (Lib.) Sacc., 
Olla millepunctata (Lib.) Svrček, Olla scrupulosa (P. Karst.) Svrček, Olla scrupulosa var. obscura Svrček, Olla scrupulosa (P. Karst.) Svrček, var. scrupulosa, Peziza carmichaelii W. Phillips, Peziza cirrhata P. Crouan \& H. Crouan, Peziza elaphines Berk. \& Broome, Peziza millepunctata Lib., in Roumeguère, Peziza scrupulosa (P. Karst.) P. Karst., Pezizella dematiicola Feltgen, Pezizella millepunctata (Lib.) Rehm, Pezizella scrupulosa (P. Karst.) Rehm, Phialea carmichaelii (Berk.) Sacc., Pseudohelotium elaphines (Berk. \& Broome) Sacc., Pseudohelotium millepunctatum (Lib.) Sacc., Pseudohelotium scrupulosum (P. Karst.) Sacc., Pseudohelotium scrupulosum var. carpini Sacc., seudohelotium scrupulosum var. caulium Sacc., Pseudohelotium scrupulosum (P. Karst.) Sacc., var. scrupulosum, Pyrenopeziza grisella (Cooke \& W. Phillips) Boud., Pyrenopeziza grisella (Cooke \& W. Phillips) Boud., var. grisella, Pyrenopeziza grisella var. ilicis (Feltgen) Boud., Sclerotinia ulmariae Velen., Tapesia dematiicola (Feltgen) Boud., Trichopeziza cirrhata (P. Crouan \& H. Crouan) Sacc., Trichopeziza grisella (Cooke \& W. Phillips) Sacc., Trichopeziza grisella (Cooke \& W. Phillips) Sacc., f. grisella, Trichopeziza grisella f. ilicis
(Feltgen) Sacc. \& D. Sacc., Unguicularia cirrhata (P. Crouan \& H. Crouan) Le Gal, Unguicularia millepunctata (Lib.) Dennis, Unguicularia scrupulosa (P. Karst.) Höhn., Unguicularia ulmariae (Velen.) Dennis, Urceola elaphines (Berk. \& Broome) Quél., Urceolella cirrhata (P. Crouan \& H. Crouan) Boud., Urceolella elaphines (Berk. \& Broome) Boud., Urceolella scrupulosa (P. Karst.) Boud., Urceolella scrupulosa var. carpini (Sacc.) Boud., Urceolella scrupulosa var. caulia (Sacc.) Boud., Urceolella scrupulosa (P. Karst.) Boud., var. scrupulosa]

Macroscopic and microscopic features: Apothecia 0.1-0.3 mm in diameter, urceolate, sessile, whitish to pale greyish when fresh, whitish gray to pale olivaceous gray when dry. Hymenial surface smooth, gray-whitish to ocher (Figure 3a). Asci 30-40 × 4-7 $\mu \mathrm{m}$, clavate, eight spored. Paraphyses filiform, sometimes branched in basal part (Figure 3b). Ascospores $5-7,5 \times 1-1,8 \mu \mathrm{m}$, ellipsoid, sometimes clavateellipsoid, smooth, hyaline (Figure 3c).

Specimen examined: GaziantepNurdağı, Gökçedere village, roadside, on dead Rubus L. sp. stem, $37^{\circ} 09^{\prime} \mathrm{N}-36^{\circ} 42^{\prime} \mathrm{E}, 485 \mathrm{~m}$, 07.03.2014, K.8500.

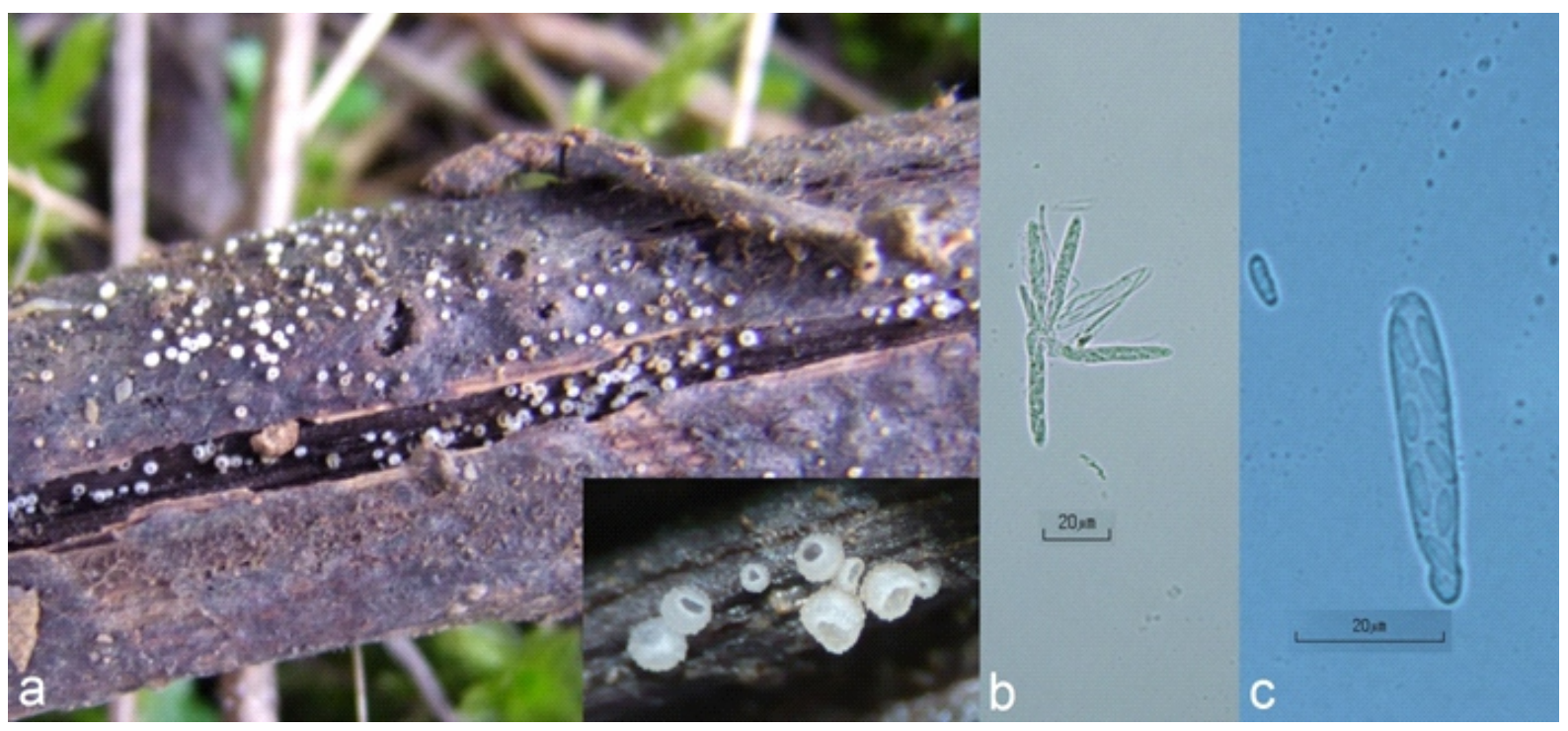

Figure 3. Hyalopeziza millepunctata: a. ascocarps, b. asci and paraphyses, c. ascospores in an ascus 


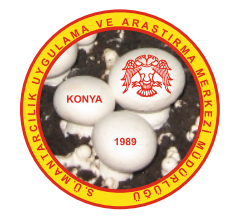

\section{Lasiobelonium variegatum (Fuckel)} Raitv.

Syn: [Atractobolus variegatus (Fuckel) Kuntze, Dasyscyphus variegatus Fuckel, Lachnum variegatum (Fuckel) Rehm, Lachnum variegatum (Fuckel) Rehm f. variegatum]

Macroscopic and microscopic features: Apothecia $0.5-1.5 \mathrm{~mm}$ in diameter, sessile, cupulate, external surface clearly hairy, hairs are dense towards the margin and loose towards the base. Hymenial surface smooth, cream to gray (Figure 4a). Asci 60-70 × 6-7 $\mu \mathrm{m}$ cylindrical, eight spored. Paraphyses 60-90 $\times 2$ $4 \mu \mathrm{m}$, somehow longer than asci, cylindrical with attenuated top (Figure 4b). Ascospores 10-16 $\times$ 2-2,5 $\mu \mathrm{m}$, oblong, slightly curved, some with small guttules towards the ends (Figure 4c).

Specimen examined: Gaziantep -Yavuzeli, Halilbaşlı village, on Populus L. sp. stump, $37^{\circ} 16^{\prime} \mathrm{N}-37^{\circ} 31^{\prime} \mathrm{E}, 560 \mathrm{~m}, 02.10 .2014$, K.10489; Sarıbuğdaylı village, $37^{\circ} 17^{\prime} \mathrm{N}-$ $37^{\circ} 31^{\prime} \mathrm{E}, 560 \mathrm{~m}, 16.03 .2014$, K. 8676.

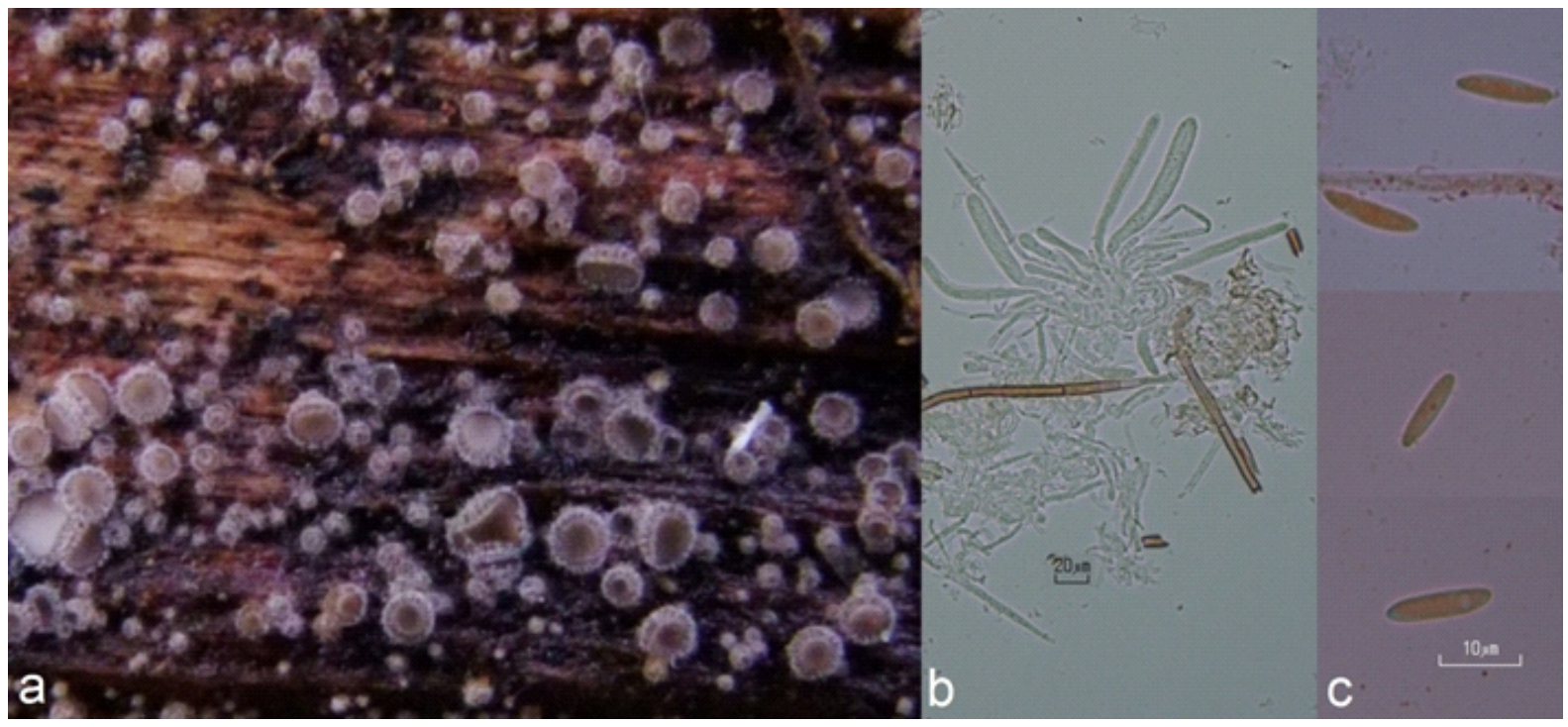

Figure 4. Lasiobelonium variegatum: a. ascocarps, b. asci and paraphyses, c. ascospores

Rodwayella citrinula (P. Karst.) Spooner

Syn: [Calycina flexuosa (Massee) Kuntze, Helotium citrinulum P. Karst., Helotium citrinulum P. Karst. var. citrinulum, Helotium citrinulum var. seaveri Rehm, Helotium flexuosum Massee, Hymenoscyphus citrinulus (P. Karst.) J. Schröt., Mollisiella citrinula (P. Karst.) Boud., Pezizella citrinula (P. Karst.) Sacc.]

Macroscopic and microscopic features: Apothecia $0.5-3 \mathrm{~mm}$ in diameter, slightly convex to flat or disc shaped, sessile, smooth or somehow pruinose, pale ocher yellow, lighter at receptacle, hymenium smooth or finely puberulent, lemon to yellowish white (Figure 5a). Asci 40-60 × 4.5-6 $\mu \mathrm{m}$, cylindric to cylindric-clavate, eight spored. Paraphyses cylindric with slightly swollen apex (Figure $5 b$ ). Ascospores 7.5-12 × 1.5-2.5 $\mu \mathrm{m}$ elliptic-fusoid, elliptic-clavate, straight or slightly bent, hyaline, smooth, sometimes constricted at the centre (Figure 5c).

Specimen examined: Gaziantep -Nurdağı, Olucak village, mixed forest, roadside, on dead herbs, $37^{\circ} 10^{\prime} \mathrm{N}-36^{\circ} 40^{\prime} \mathrm{E}, 950$ $\mathrm{m}, 10.04 .2015$, K.11682. 

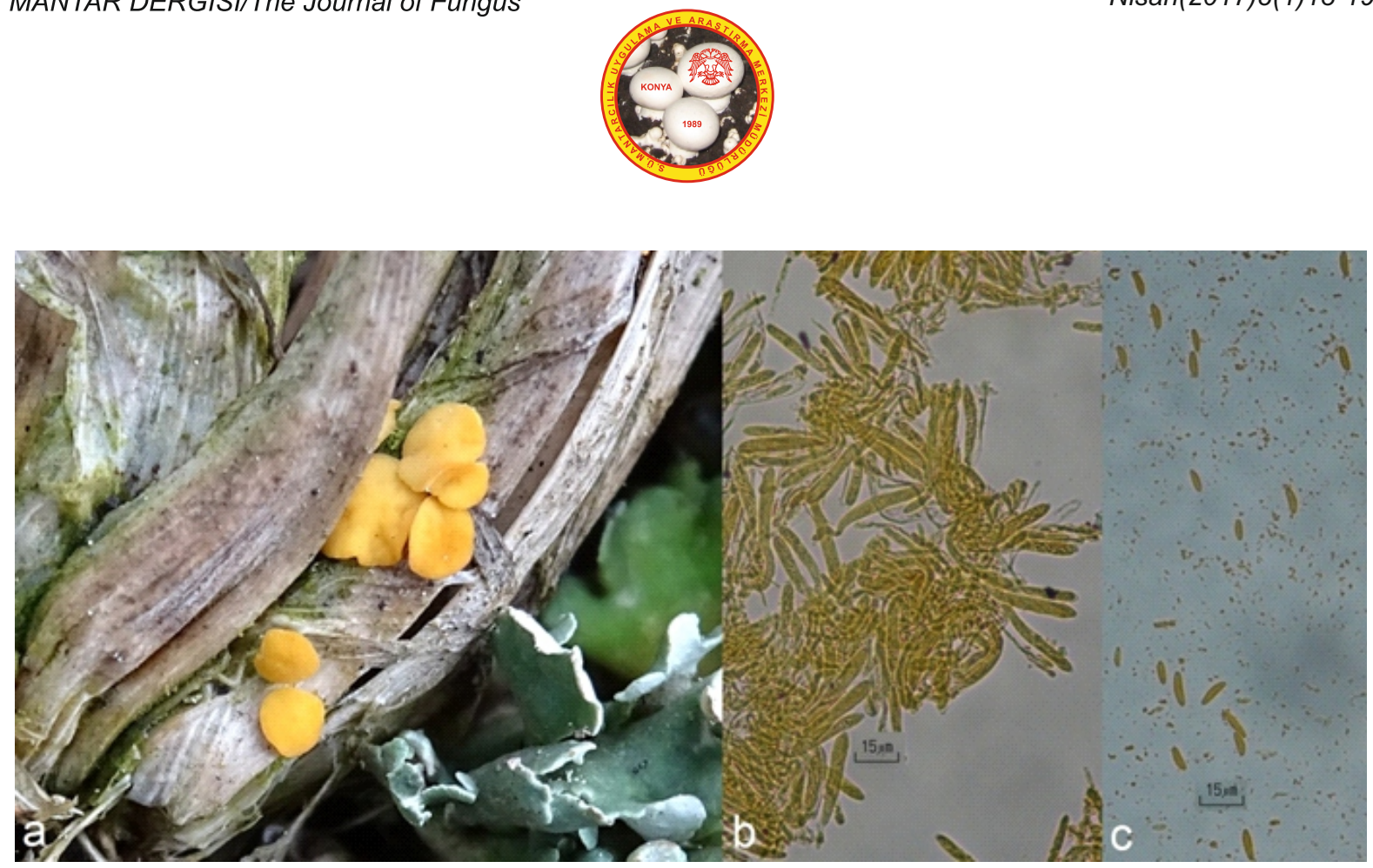

Figure 5. Rodwayella citrinula: a. ascocarps, b. asci and paraphyses, c. ascospores

\section{Discussion}

New contributions were made to the mycobiota of Turkey with the addition of five members of Hyaloscyphaceae. Among them Calycina conorum (Rehm) Baral, Discocistella grevillei (Berk.) Svrček, Hyalopeziza millepunctata (Lib.) Raitv. and Rodwayella citrinula (P. Karst.) Spooner are the first members of the genera Calycina Nees ex Gray, Discocistella Svrček, Hyalopeziza Fuckel and Rodwayella Spooner respectively, while
Lasiobelonium variegatum (Fuckel) Raitv. is the second member of the genus Lasiobelonium Ellis \& Everh. in Turkey (Kaya et al., 2015). As a result, the genera and the total taxa number of Hyaloscyphaceae in Turkey increased to 10 and 15 respectively.

\section{Acknowledgements}

The authors would like to thank TÜBITAK for supporting the Project (212T112) financially.

\section{References}

Akata I., Çetin B., Işıloğlu M. Macrofungi of Ankara-Kızılcahamam Soğuksu National park. The Herb Journal of Sytematic Botany, 16(2): 177-188 (2009a).

Akata I., Doğan H.H., Körüklü T., İşlek, C. Macrofungi of Ankara University Tandoğan campus. Kafkas University Journal of Science, 2(1): 15-19 (2009b).

Alkan, S., Kaşık, G. \& Aktaş, S. Macrofungi of Derebucak district (Konya, Turkey). Turkish Journal of Botany, 34: 335-350 (2010).

Breitenbach J., Kränzlin F. Fungi of Switzerland. Volume 1: Ascomycetes. Luzern, Switzerland: Verlag Mykologia (1984).

Cannon PF, Kirk PM. Fungal Families of the World. Wallingford, UK: CAB International (2007).

Cheype JL. Contribution à la connaissance des champignons de la haute vallée de l'Arve (Haute-Savoie) 3e note: deux discomycètes inoperculés « au poil ». Bull. Mycol. Bot. Dauphiné-Savoie, 173: 2936 (2004).

Doğan H.H., Öztürk C. Macrofungi and their distribution in Karaman Province, Turkey. Turkish Journal of Botany, 30: 193-207 (2006). 
Friebes G, Wendelin I. Wer suchet, der findet: Seltene und interessante Ascomycota vom Jägerberg (Steiermark, Österreich). Joannea Botanik, 12: 5-38 (2015).

Hairaud M. Note sur deux espèces intéressantes. Ascomycete.org, 2(2): 11-13 (2010).

Han J.G., Sung G.H. Shin H.D. Proliferodiscus inspersus var. magniascus and Rodwayella citrinula, Two Unrecorded Taxa of Hyaloscyphaceae (Tribe Arachnopezizeae) in Korea. Mycobiology, 41: 86-91 (2014).

Kaşık G., Doğan H.H., Öztürk C., Aktaş, S. New records of Ascomycetes for Turkish mycoflora. Selçuk Üniversitesi Fen Edebiyat Fakültesi Fen Dergisi, 20: 75-81 (2002).

Kaya A., Karacan I.H., Uzun Y. Three Phragmites Adans. inhabiting fungi, new for Turkey. Biological Diversity and Conservation, 8(1):143-146 (2015).

Kirk PM, Cannon PF, Minter DW, Stalpers JA. Dictionary of the Fungi. 10th ed. Wallingford, UK: CAB International (2008).

Öztürk Ö., Doğan H.H., Yıldırımlı, Ş. Macrofungi of Eldivan dağ (Çankııı). The Herb Journal of Systematic Botany, 17(2): 141-154 (2010).

Raitviir A. The Genus Lasiobelonium. Scripta Mycologica, 9: 99-132 (1980).

Raitviir A., Galán R. Notes on Spanish glassy-haired Hyaloscyphaceae. Sydowia, 45(1): 34-54 (1993).

Sesli E., Denchev CM. Checklists of the myxomycetes, larger ascomycetes, and larger basidiomycetes in Turkey. 6th edn. Mycotaxon Checklists Online (http://www.mycotaxon.com/resources/checklists/sesli-v106-checklist.pdf): 1-136 (2014).

Sümer, S. Wood-decaying fungi in the western Black Sea Region of Turkey, especially in and around Bolu Province. İstanbul Üniversitesi Orman Fakültesi Yayınları, İstanbul (1982).

Uzun Y., Kaya A., Karacan I.H., Kaya Ö.F., Yakar S. Macromycetes determined in Islahiye (Gaziantep/Turkey) district. Biological Diversity and Conservation, 8(3): 209-217 (2015). 